\title{
Thought disorder in schizophrenia is associated with both executive dysfunction and circumscribed impairments in semantic function
}

\author{
JOHN STIRLING ${ }^{1 *}$, JONATHAN HELLEWELL ${ }^{2}$, ANDREW BLAKEY ${ }^{3}$ \\ AND WILLIAM DEAKIN ${ }^{4}$ \\ ${ }^{1}$ Department of Psychology and Speech Pathology, Manchester Metropolitan University, Manchester, UK; \\ ${ }^{2}$ Moorside Unit, Department of Psychiatry, Trafford General Hospital, Manchester, UK; ${ }^{3}$ Department of \\ Psychiatry, Macclesfield General Hospital, Macclesfield, Cheshire, UK $;{ }^{4}$ Neuroscience and Psychiatry Unit, \\ Stopford Building, University of Manchester, Manchester, UK
}

\begin{abstract}
Background. Formal thought disorder (FTD) has long been regarded as a key sign of schizophrenia but little is known about its origins or aetiology. One suggestion is that it is directly related to disordered language functioning; a second is that it is a reflection of poor neurocognitive functioning. A current model posits that it is related to a combination of executive dysfunction and impaired semantic processing.
\end{abstract}

Method. To examine these alternative ideas, a heterogeneous group of 30 patients, all carrying a diagnosis of schizophrenia, and 18 non-patient controls completed a series of neurocognitive and psycholinguistic tests, and a clinical review that, inter alia, permitted assessment of thought disorder (TD) using the Thought, Language and Communication Scale (TLC).

Results. Patients generally performed at a lower level on most components of the test battery, but there was little evidence of a relationship between TD and syntactic psycholinguistic function. However, schizophrenic patients manifesting higher levels of TD performed at a lower level on tests sensitive to executive dysfunction and semantic impairments.

Conclusions. The origins of TD seem more closely linked to deficits in executive functioning and semantic processing than to impairments in other language functions or general cognition.

\section{INTRODUCTION}

Disordered thinking and unusual patterns of speech have come to be regarded by many as sine qua non of schizophrenia (Bleuler, 1950). Clinically defined formal thought disorder (FTD), although relatively uncommon, is certainly recognized as an important feature of the illness, but its origins, and even its precise nature, remain unclear. Early

\footnotetext{
* Address for correspondence: Dr John Stirling, Department of Psychology and Speech Pathology, Manchester Metropolitan University, Hathersage Road, Manchester, M13 OJA, UK. (Email: j.stirling@mmu.ac.uk)
}

neuropsychological approaches characterized FTD as a manifestation of either impaired executive function (McGrath, 1991; Anand et al. 1994) or impaired linguistic function (Tamlyn et al. 1992; Mortimer et al. 1995), although the evidence in support of these hypotheses is mixed (Kerns \& Berenbaum, 2002).

More recently, Goldberg et al. (1998) and Oh et al. (2002) have independently concluded that thought disorder (TD) is related to rather specific underlying semantic impairments. In the former study, schizophrenic patients with higher TD ratings evinced signs of impaired 'semantic efficiency' (semantic fluency corrected 
for phonological fluency) and word-picture matching (Peabody picture vocabulary test; Dunn \& Dunn, 1981). In the latter report, the authors described six patients with marked TD who differed from seven with little or no TD on a range of tests designed to 'tap' receptive and expressive semantic functions. Oh et al. (2002) observed that, although poor performance on their test battery was associated with general intellectual impairment rather than TD per se, patients with marked TD nevertheless showed evidence of expressive semantic disturbances regardless of their level of general cognitive function.

Rodriguez-Ferrera et al. (2001) and Barrera et al. (2005) have sought to clarify the relationships between TD, neurocognitive and psycholinguistic functions. Schizophrenic patients with and without TD, and (in the Barrera et al. study) a non-patient control group, have been evaluated using a range of both semantic and cognitive/executive function tests. In the former study, TD was associated with general intellectual impairment and semantic deficits in comprehension and picture description. In the latter, higher TD patients were impaired on all measures of executive function but on only one of four semantic tests, in comparison to low TD patients and controls. Both studies therefore provide strong support for a dysexecutive hypothesis of the origins of TD in schizophrenia (McGrath, 1991) and some additional evidence implicating a higher-level semantic associative impairment.

Andreasen (1979) developed the Thought, Language and Communication Scale (TLC) as an objective, quantitative measure of TD, partly in response to long-standing concerns about definitional clarity. Several of the studies mentioned above (Goldberg et al. 1998; Rodriguez-Ferrera et al. 2001; Oh et al. 2002) have used the TLC to assess TD and we have followed suit. By recruiting a mixed group of schizophrenic patients to be assessed on a battery of tests tapping frontal/executive function, more general neurocognitive functions and syntactic and semantic psycholinguistic functions, our correlational approach enabled us to investigate the strength of relationship between TD and impaired neurocognitive and linguistic function in this heterogeneous sample.

\section{METHOD}

\section{Participants}

Thirty patients were recruited to take part in the study on the basis that they were considered by their psychiatrist to have schizophrenia; were aged between 16 and 45 at the time of initial diagnosis; and there was no evidence of current drug misuse, organic brain disease, or marked intellectual deficit. Information obtained from case-note review, discussion with the patient's psychiatrist, and clinical interview was collated. Comparisons with published guidelines confirmed that all patients met DSM-IV criteria for schizophrenia (APA, 1994). Eight were inpatients on an acute ward of a hospital department of psychiatry at the time of testing; three were recruited from a day hospital; and 19 attended the same hospital as out-patients. The mean age of this group was 34.33 years (s.D. $=10 \cdot 39)$. There were 18 males and 12 females. The average duration of treated illness was 97 months. Twenty-eight of the 30 patients were receiving neuroleptic medication at the time of testing [average daily dose (CPZ equivalents): $573 \mathrm{mg}$ ].

Eighteen control subjects were recruited from among hospital and university personnel. These individuals stated that they had not previously had, or been treated for, psychological illness, and all responded negatively to a series of screening questions compiled to identify individuals with a current psychological disorder. The mean age of this group was $36 \cdot 22$ years (S.D. $=9 \cdot 32$ ).

\section{Clinical assessments}

All patients completed a detailed clinical interview lasting approximately 1 hour. From this it was possible to rate participants in respect of current symptomatology on the Positive and Negative Syndrome Scale (PANSS; Kay et al. 1986) and the TLC. The former was used to derive a score of 'thought disturbance' and the latter was scored in terms of total TD and global TD rating.

\section{Neuropsychological assessments}

All respondents completed a battery comprising semantic and syntactic psycholinguistic tests, measures of executive function and tests of more general neurocognition, administered by A.B., 
usually within a day or two of the clinical interview (in the case of patients). Order was standardized to ensure best use of time, and testing took approximately 90 minutes.

\section{Semantic tests}

Graded Naming Test (GNT) (McKenna \& Warrington, 1983)

Thirty progressively more obscure (i.e. less frequent) line drawings of animals and objects must be identified (named) by the respondent.

\section{The Pyramids and Palm Trees Test (PPT) (Howard \& Patterson, 1992)}

This test comprises 52 triads of items, in which one (shown at the top of the page) has to be matched to one of the others (shown below). This test assesses a person's ability to access detailed semantic information from pictures.

\section{Speed and Capacity of Language Processing} (SCOLP) (Baddeley et al. 1992)

This test has two elements. The 'speed of comprehension test' (silly sentences test) comprises a series of grammatically correct statements about the world, of which only half make sense. The respondent assesses the validity of as many as possible within a 2-minute time period. The 'spot the word test' comprises 60 word pairs; one a proper word and the other a nonsense word (e.g. violin-broft). The respondent must select the real word, guessing if necessary.

\section{Semantic fluency (Spreen \& Benton, 1969) and} 'semantic efficiency' (Goldberg et al. 1998)

In the semantic fluency test, the respondent produces as many exemplars as possible of animals, countries, and fruits (60 seconds for each category). A score of 'semantic efficiency' (retrieval of semantic knowledge from memory corrected for the more executive task of 'phonological fluency'; see below) is derived by subtracting phonological fluency score from semantic fluency score.

\section{The Quick Test (QT) (Ammons \& Ammons, 1962)}

This test is more commonly used as a proxy for current IQ but does, in effect, 'tap' semantic matching, and we have used it in this way.
Standard instructions and procedure for form 1 were followed. Respondents see a card showing four cartoon drawings. The tester reads from a list of 50 words of increasing complexity and respondents point to the picture most closely related to the word. The test is terminated if/when the respondent makes six consecutive errors.

\section{Syntactic tests}

Test for the Reception of Grammar (TROG) (Bishop, 1989)

This test measures the understanding of grammatical constructs using 80 four-choice items, for each of which the respondent selects a picture corresponding to the word or phrase spoken by the tester. It is divided into 20 fouritem blocks arranged in increasing difficulty. A block is only 'passed' if all four items have been correctly dealt with. A respondent's score is the number of blocks passed.

\section{Token test (DeRenzi \& Vignolo, 1962)}

This test measures the comprehension of commands varying in linguistic complexity. The test materials consist of circular and square shapes of various sizes and colours. The respondent has to follow commands ranging from simple ones such as 'Show me a circle', to more complex conditional ones such as 'If there is a black circle pick up the red square'. Performance is assessed in terms of the number of instructions followed correctly.

\section{Reporter's test (DeRenzi \& Ferrari, 1978)}

This test measures expressive syntactic skills by requiring the respondent to act as a 'reporter' of the tester's actions (on the token test) in such a way that a third party could reproduce those actions exactly. The complexity of actions, and thus the speech required to accurately and succinctly describe them, increases to include up to six elements (e.g. 'Touch the large white square and the small yellow circle'). Performance is assessed in terms of accuracy in reporting the requisite actions.

\section{Tests of executive function}

Stroop test (Stroop, 1935)

In the word task, the respondent is shown a card with the words BLUE, RED, GREEN, and TAN 
(printed in contrasting colours) arranged in four 30 -word columns, to be read as quickly as possible ignoring their colours. In the colour task, a similar set of words is shown to the respondent who must now identify the colour in which each word is printed. The Stroop effect is the difference (in seconds) between the times to complete the two tasks.

\section{Trail-making test (Reitan, 1958)}

In form $\mathrm{A}$, the respondent draws a line (as quickly as possible) to connect together, in ascending order, 25 numbered circles scattered apparently randomly on a single page. Form B comprises 13 numbered circles (1 to 13) and 12 lettered circles (A to L) scattered randomly on a page, to be connected by a continuous line linking 1 to $\mathrm{A}, 2$ to $\mathrm{B}, 3$ to $\mathrm{C}$, and so on. The Trails score is the difference (in seconds) in completing tests $\mathrm{A}$ and $\mathrm{B}$.

\section{Design fluency (Jones-Gotman \& Milner, 1977)}

This tests fluency in a non-verbal mode. In the fixed condition, respondents must generate as many different designs as possible using just four lines (4 minutes allowed). In the free condition, they should generate as many drawings (doodles and nonsense drawings but not scribbles or nameable geometric shapes) as possible in a 5-minute period. Fluency is assessed in terms of the total number of exemplars and the number of novel exemplars produced.

\section{Tower of London test (Shallice, 1982)}

This test taps higher-level planning faculties, by requiring respondents to move a set of three differently coloured beads from a consistent starting position on three short poles to a specified target pattern, in the minimum number of moves. Puzzles require between two and fives moves for resolution. The more moves required, the greater the load placed on generation of, and search for, optimal solutions skills usually associated with frontal lobe function.

\section{Phonological fluency (Spreen \& Benton, 1969)}

For phonological fluency the respondent should produce as many words as possible beginning with the letters S, A, and F (60 seconds each).
This test is thought to depend on the integrity of the left frontal lobe. The score is the total number of words generated excluding repetitions in 3 minutes.

\section{Tests of more general cognitive function}

Forward and reverse digit span (Wechsler, 1987)

Forward digit span assesses short-term memory. Respondents must repeat progressively longer sequences of digits read aloud to them at a rate of one digit per second. Span is defined as the longest string of digits correctly recalled at least once. Reverse span is administered in similar fashion but respondents must now reproduce the sequence in the reverse of the presentation order. This task involves manipulation of information as well as simple reiteration of it, and is often cited as a measure of working memory (Stirling et al. 1997).

\section{National Adult Reading Test (NART) (Nelson \& O'Connell, 1978)}

This test provides an estimate of pre-morbid IQ. Respondents attempt to read aloud from a list of 50 words of increasing obscurity and non-standard phonetic structure, typed on a card. An error is recorded for each incorrect pronunciation. Correct pronunciation depends on prior encounter with the words, and in nonclinical samples is highly correlated with other measures of verbal IQ.

\section{Raven's Standard Progressive Matrices (RPM) (Raven, 1960)}

The test comprises five sets of 12 problems, each consisting of a pattern with part removed, and six alternative parts, each of which fits into the space, but only one of which completes the pattern. The respondent's non-verbal IQ is determined from the number of correctly solved problems using standardized conversion tables. An estimate of IQ decline was derived by subtracting the RPM score from the NART score.

\section{Information processing speed (Coghlan, 1985)}

The respondent works through a list of pairs of numbers; each pair comprising a fourand a five-digit number. The five-digit number is made up of the same four digits as the 
Table 1. Patients versus controls: age, pre-morbid and current IQ/IQ decline

\begin{tabular}{|c|c|c|c|c|c|}
\hline & \multicolumn{2}{|c|}{ Patients } & \multicolumn{2}{|c|}{ Controls } & \multirow[b]{2}{*}{$p$ value ${ }^{\mathrm{a}}$} \\
\hline & Mean & (S.D.) & Mean & (S.D.) & \\
\hline$N$ & 30 & & 18 & & \\
\hline Age & $34 \cdot 33$ & & $36 \cdot 22$ & & N.S. \\
\hline Estimated pre-morbid IQ (NART) & $102 \cdot 70$ & $(7 \cdot 84)$ & $103 \cdot 17$ & $(11.69)$ & N.S. \\
\hline Current IQ (RPM) & 93.60 & $(10 \cdot 11)$ & $102 \cdot 00$ & $(13 \cdot 46)$ & 0.018 \\
\hline IQ decline (NART - RPM) & $9 \cdot 10$ & $(5 \cdot 70)$ & $1 \cdot 17$ & $(4 \cdot 51)$ & $<0.001$ \\
\hline
\end{tabular}

a Student's independent $t$ test (two-tailed).

NART, National Adult Reading Test; RPM, Raven's Standard Progressive Matrices.

Table 2. Patients versus controls: neurocognitive and executive functions

\begin{tabular}{|c|c|c|c|c|c|}
\hline & \multicolumn{2}{|c|}{ Patients } & \multicolumn{2}{|c|}{ Controls } & \multirow[b]{2}{*}{$p$ value $^{\mathrm{a}}$} \\
\hline & Mean & (S.D.) & Mean & (s.D.) & \\
\hline Design fluency (fixed) & $7 \cdot 87$ & $(11 \cdot 33)$ & $14 \cdot 00$ & $(2 \cdot 87)$ & $0 \cdot 030$ \\
\hline Design fluency (free) & $7 \cdot 20$ & $(3 \cdot 71)$ & $16 \cdot 00$ & $(5 \cdot 15)$ & $<0 \cdot 001$ \\
\hline Total design fluency (novelty) & $15 \cdot 06$ & $(12.91)$ & $30 \cdot 00$ & $(7 \cdot 54)$ & $<0 \cdot 001$ \\
\hline Phonological fluency (FAS) & $22 \cdot 97$ & $(7 \cdot 54)$ & $41 \cdot 33$ & $(4 \cdot 54)$ & $<0 \cdot 001$ \\
\hline Stroop effect (seconds) & $123 \cdot 20$ & $(62 \cdot 52)$ & $58 \cdot 12$ & $(11 \cdot 258)$ & $<0.001$ \\
\hline Trails B - A (seconds) & $66 \cdot 48$ & $(30 \cdot 09)$ & 33.99 & $(8 \cdot 53)$ & $<0 \cdot 001$ \\
\hline Tower of London (trials completed) & $7 \cdot 47$ & $(2 \cdot 36)$ & $9 \cdot 44$ & $(1 \cdot 76)$ & $0 \cdot 004$ \\
\hline Forward digit span & $8 \cdot 46$ & $(2 \cdot 11)$ & $9 \cdot 67$ & $(1 \cdot 18)$ & $0 \cdot 015$ \\
\hline Reverse digit span & $5 \cdot 53$ & $(2 \cdot 24)$ & $9 \cdot 33$ & $(2 \cdot 17)$ & $<0 \cdot 001$ \\
\hline $\begin{array}{l}\text { Coghlan information processing } \\
\text { (motor speed adjusted) }\end{array}$ & $50 \cdot 00$ & $(13.98)$ & $68 \cdot 67$ & $(8 \cdot 87)$ & $<0 \cdot 001$ \\
\hline
\end{tabular}

a Student's independent $t$ test (two-tailed).

first number, plus an extra digit, which the subject has to identify. Four minutes is allowed, and the measure obtained (corrected for motor speed) reflects speed of information processing.

\section{Data analysis}

Analysis was undertaken using SPSS version 12.0 (SPSS Inc., Chicago, IL, USA). Data were reviewed for 'normality' and transformed if necessary prior to analysis (tables show untransformed values). Patients and controls were compared using a series of independent $t$ tests. Within the patient group, bivariate correlations were calculated for total TD with both the neuropsychological and semantic/syntactic tests. These relationships were further explored in a series of regression analyses setting total TD as the dependent variable and the tests in the relevant four domains as independent variables.

\section{RESULTS}

\section{Comparison of patients and controls}

\section{Age, IQ and IQ decline (Table 1 )}

There was no significant difference between groups in respect of age or estimated premorbid IQ (NART). There was a significant difference in terms of current IQ (RPM) and in IQ decline (NART - RPM).

Neurocognitive and executive function (Table 2)

These data provide evidence of a consistent and widespread deficit in our patient group, who performed at a significantly lower level on our measures of fluency, information processing, short-term memory and frontal/executive function (Stroop effect, Trails B-A, Tower of London).

\section{Semantic and syntactic function (Table 3)}

These data provide evidence of a generally poorer performance on all psycholinguistic 
Table 3. Patients versus controls: semantic and syntactic functions

\begin{tabular}{|c|c|c|c|c|c|}
\hline & \multicolumn{2}{|c|}{ Patients } & \multicolumn{2}{|c|}{ Controls } & \multirow[b]{2}{*}{$p$ value $^{\mathrm{a}}$} \\
\hline & Mean & (S.D.) & Mean & (S.D.) & \\
\hline Semantic fluency & $42 \cdot 73$ & $(9 \cdot 41)$ & $51 \cdot 39$ & $(4 \cdot 98)$ & $<0 \cdot 001$ \\
\hline Speed of comprehension test ${ }^{b}$ & $5 \cdot 30$ & $(2 \cdot 26)$ & $10 \cdot 72$ & $(2 \cdot 89)$ & $<0.001$ \\
\hline Spot the word test & $45 \cdot 07$ & $(6 \cdot 40)$ & $48 \cdot 83$ & $(4.99)$ & 0.038 \\
\hline Quick test & $92 \cdot 33$ & $(10 \cdot 56)$ & $102 \cdot 61$ & $(12 \cdot 70)$ & $0 \cdot 004$ \\
\hline Graded Naming Test & $18 \cdot 10$ & $(4 \cdot 05)$ & $20 \cdot 28$ & $(3 \cdot 73)$ & N.S. \\
\hline Pyramids and palm trees test & $50 \cdot 83$ & $(1 \cdot 15)$ & $51 \cdot 19$ & $(0 \cdot 91)$ & N.S. \\
\hline Token test ${ }^{\mathrm{c}}$ & $76 \cdot 91$ & $(1 \cdot 30)$ & $77 \cdot 74$ & $(0 \cdot 65)$ & N.S. \\
\hline Reporter's test ${ }^{\mathrm{c}}$ & $59 \cdot 00$ & $(1 \cdot 39)$ & $59 \cdot 61$ & $(0 \cdot 91)$ & N.S. \\
\hline TROG $^{\mathrm{d}}$ & $17 \cdot 60$ & $(1 \cdot 25)$ & $19 \cdot 67$ & $(0 \cdot 67)$ & $<0 \cdot 001$ \\
\hline
\end{tabular}

a Student's independent $t$ test (two-tailed).

${ }^{\mathrm{b}}$ Scaled score; ${ }^{\mathrm{c}}$ weighted; ${ }^{\mathrm{d}}$ number.

TROG, Test for the Reception of Grammar.

tests. However, significant differences between patients and controls were limited to the reporter and TROG tests, and both components of the SCOLP.

\section{Relationship between TD, neurocognitive and psycholinguistic profile and IQ within the patient group}

[Thought disturbance score derived from the PANSS (items P2, P3, P5 and G9 summed) correlated highly significantly with TLC total TD score $(r=0.733, p=0.001)$. Thus, to avoid duplication, the following analyses are restricted to TLC-derived scores.]

In the neurocognitive and executive domains, total TD correlated inversely with reverse digit span $(r=-0.407, p=0.026)$ and information processing speed $(r=-0.401, p=0.028)$, positively with both Trail tests (Trails A : $r=0.528$, $p=0.003$; Trails B: $r=0.543, p=0.002$ ), and crucially with the difference score between Trails A and B $(r=0.432, p=0 \cdot 017)$. Total TD correlated inversely with current IQ (RPM: $r=-0.399, \quad p=0.029)$, and also correlated significantly with three semantic tests - fluency $(r=-0.502, \quad p=0.005), \quad$ GNT $\quad(r=-0.418$, $p=0 \cdot 21)$ and QT $(r=-0 \cdot 404, p=0 \cdot 027)$.

Following Goldberg et al. (1998) and in similar fashion to Barrera et al. (2005), who used the Comprehensive Assessment of Symptoms (CASH; Andreasen, 1987) rather than the TLC to derive TD scores, we divided patients into higher and lower TD groups on the basis of whether their TLC (global) rating was 2 or more (higher TD; $n=11$ ) or less than
2 (lower/absent TD; $n=19$ ). The higher TD group were poorer than the lower group on the Trails test $(t=2.36, p=0.025)$, information speed $(t=2 \cdot 29, p=0 \cdot 029)$ and reverse digit span $(t=2 \cdot 55, p=0 \cdot 017)$, and marginally poorer for design fluency novelty $(t=1.91, p=0.06)$ and the Stroop effect $(t=2 \cdot 00, p=0 \cdot 055)$. In the psycholinguistic domain, the two groups differed significantly in respect of semantic fluency $(t=3.05, p=0.006)$ and QT $(t=2 \cdot 34, p=0.03)$, but not in respect of semantic efficiency (semantic fluency - phonological fluency) or any of the syntactic measures. These data are shown in Table 4.

The relationships between total TD and other variables were further examined using multiple regression. Separate stepwise analyses were computed, with total TD as the dependent variable, for each domain of interest. In the general neurocognitive domain, with forward and reverse digit span, current and pre-morbid IQ and information processing speed as independent variables, reverse digit span emerged as the only predictive variable $(t=2 \cdot 61, p=0 \cdot 01)$. In respect of executive function, with Trails difference, Tower of London, design and phonological fluency and Stroop effect as independent variables, Trails difference was the only predictive variable $(t=2 \cdot 32, p=0.03)$. In the semantic domain, with GNT, PPT, semantic fluency, both elements of the SCOLP and QT as independent variables, fluency was the best/only predictor $(t=2.73, p=0.01)$. Total TD was not predicted by any of the syntactic psycholinguistic assessments. 
Table 4. Higher $(\geqslant 2)$ versus lower $(<2)$ TD groups: selected neurocognitive, executive and psycholinguistic measures

\begin{tabular}{|c|c|c|c|c|c|}
\hline & \multicolumn{2}{|c|}{ Moderate to high TD $(n=11)$} & \multicolumn{2}{|c|}{ Low/absent TD $(n=19)$} & \multirow[b]{2}{*}{$p$ value $^{\mathrm{a}}$} \\
\hline & Mean & (S.D.) & Mean & (S.D.) & \\
\hline Trails B - A (seconds) & $82 \cdot 34$ & $(34 \cdot 69)$ & $57 \cdot 30$ & $(23 \cdot 38)$ & $0 \cdot 025$ \\
\hline $\begin{array}{l}\text { Coghlan information processing } \\
\text { (motor speed adjusted) }\end{array}$ & $43 \cdot 55$ & $(9 \cdot 18)$ & $53 \cdot 74$ & $(15 \cdot 09)$ & $0 \cdot 029$ \\
\hline Reverse digit span & $4 \cdot 45$ & $(1 \cdot 12)$ & $6 \cdot 16$ & $(2 \cdot 50)$ & 0.017 \\
\hline Total design fluency (novelty) & $9 \cdot 36$ & $(3 \cdot 91)$ & $18 \cdot 36$ & $(15 \cdot 24)$ & $0 \cdot 066$ \\
\hline Stroop effect (seconds) & $151 \cdot 79$ & $(77 \cdot 30)$ & $106 \cdot 65$ & $(46 \cdot 77)$ & $0 \cdot 055$ \\
\hline Semantic fluency & $36 \cdot 64$ & $(8 \cdot 37)$ & $46 \cdot 26$ & $(8 \cdot 25)$ & $0 \cdot 006$ \\
\hline Phonological fluency & $20 \cdot 91$ & $(5 \cdot 30)$ & $24 \cdot 16$ & $(8 \cdot 48)$ & N.S. \\
\hline $\begin{array}{l}\text { Semantic efficiency (semantic } \\
\text { fluency - phonological fluency) }\end{array}$ & $15 \cdot 72$ & $(8 \cdot 69)$ & $22 \cdot 10$ & $(12 \cdot 67)$ & N.S. \\
\hline Quick test & $86 \cdot 82$ & $(7 \cdot 72)$ & $95 \cdot 53$ & $(10 \cdot 83)$ & $0 \cdot 030$ \\
\hline Graded naming test & $16 \cdot 73$ & $(2 \cdot 68)$ & $18 \cdot 89$ & $(4 \cdot 54)$ & N.S. \\
\hline Pyramid and palm trees test & $50 \cdot 73$ & $(1 \cdot 34)$ & $560 \cdot 89$ & $(1 \cdot 04)$ & N.S. \\
\hline Speed of comprehension test ${ }^{\mathrm{b}}$ & $4 \cdot 55$ & $(2 \cdot 42)$ & $5 \cdot 74$ & $(2 \cdot 10)$ & N.S. \\
\hline Spot the word test & $42 \cdot 64$ & $(6 \cdot 65)$ & $46 \cdot 47$ & $(5 \cdot 97)$ & N.S. \\
\hline Reporter's test ${ }^{\mathrm{c}}$ & $58 \cdot 73$ & $(1 \cdot 73)$ & $59 \cdot 16$ & $(1 \cdot 16)$ & N.S. \\
\hline Token test $\mathrm{t}^{\mathrm{c}}$ & $76 \cdot 91$ & $(1 \cdot 30)$ & $77 \cdot 74$ & $(0 \cdot 65)$ & N.s. \\
\hline $\mathrm{TROG}^{\mathrm{d}}$ & $17 \cdot 27$ & $(1 \cdot 00)$ & $18 \cdot 79$ & $(1 \cdot 35)$ & N.S. \\
\hline
\end{tabular}

\footnotetext{
${ }^{a}$ Student's independent $t$ test (two-tailed).

b Scaled score; ${ }^{\mathrm{c}}$ weighted; ${ }^{\mathrm{d}}$ number

TD, Thought Disorder; TROG, Test for the Reception of Grammar.
}

\section{Semantic deficits in higher TD patients revisited}

Two semantic tests, semantic fluency and QT, differentiated patients scoring higher and lower for TD (GNT did not, despite its correlation with total TD.) We repeated these comparisons with RPM (current IQ) as covariate. Somewhat predictably, the main effect for QT (itself a proxy for current verbal IQ) was rendered nonsignificant by this $(F=0.51$, N.s. $)$. However, the main effect for semantic fluency, though reduced, remained significant $(F=4 \cdot 85, \mathrm{df}=1 \cdot 27$, $p=0.03)$. In fact this main effect also survived the addition of phonological fluency as a second covariate $(F=5 \cdot 10, \mathrm{df}=1 \cdot 26, p=0 \cdot 03)$. These results suggest that a semantic fluency deficit in higher TD patients is, to some extent, independent of current IQ or pure phonological fluency skills.

\section{Medication effects}

Correlations between neuroleptic medication at time of testing (converted into $\mathrm{CPZ}$ equivalent dosage) and each of our neuropsychological and psycholinguistic tests routinely failed to reach statistical significance. Comparison of the 10 patients who were receiving anti-cholinergic medication with the 20 who were not indicated that the former group did less well on the reporter and speed of comprehension tests $(t=2 \cdot 30, p=0.03$ and $t=2.54, p=0.02$ respectively) and better on semantic fluency $(t=2 \cdot 14$, $p=0 \cdot 04)$.

\section{DISCUSSION}

As expected, our data provide evidence of neurocognitive dysfunction among patients with a diagnosis of schizophrenia, who also performed at a significantly lower level on four psycholinguistic measures, and evinced a wide range of TD (total score range 0-22). Within the cognitive and executive function domains, TD was inversely related to reverse digit span, information processing speed and current IQ, and positively correlated to time-to-complete both Trails tests and to the difference between Trails B and A. Regression analyses identified Trails difference and reverse digit span as the best predictors of total TD. A particular role for impaired frontal lobe functioning in TD has been mooted by many authors (Harrow \& Prossen, 1978; Frith, 1992; McGrath et al. 1997; Barrera et al. 2005). Our finding that higher TD was associated with significantly worse performance on two 'frontal' tests (Trails difference and reverse digit span) and 
marginally with two more (Stroop effect and design fluency novelty, both one-tailed only) lends weight to the dysexecutive model of TD. Additionally, semantic fluency (a test that assesses semantic and executive functions) was the best of all the semantic and syntactic (psycholinguistic) measures at predicting TD. Coupled with the findings from the other multiple regression analyses, executive dysfunction, particularly that relating to failure to keep 'on track' or inhibit pre-potent responses, seems to be closely linked to increased levels of TD.

In the psycholinguistic domain, three semantic tests (GNT, QT and semantic fluency) but none of the receptive/expressive syntactic tests correlated with total TD. As mentioned above, regression analyses indicated that fluency was the only semantic or syntactic measure to predict TD. Prima facie, these findings provide additional evidence for impaired naming (GNT) and semantic access (QT and fluency) in TD. However, fluency assesses both semantic and executive functions. When the latter is controlled by subtracting phonological fluency performance to produce an index of semantic efficiency, the relationship to TD disappears; a finding at odds with Goldberg et al. (1998). However, other researchers have raised questions about the extent to which this derived measure may reflect a characteristic of impaired function in schizophrenia more generally (Gourovitch et al. 1996), or be related to symptoms other than TD (Rossell et al. 1999). We would add that test scores based on differences are susceptible to 'initial value' effects and should be treated with caution. [Goldberg et al. do not provide details of uncorrected phonological and semantic fluency rates.] With these caveats in mind, we nevertheless note that our negative findings in respect of this measure are consistent with those of Barrera et al. (2005).

Goldberg et al. and Barrera et al. independently concluded that 'higher level' semantic impairments contribute towards TD partly on the basis of the differential performance of higher and lower TD patients on different tests. We concur with this overall view despite being unable to replicate the findings of Goldberg et al. in respect of the SCOLP. Moreover, our higher TD patients could not be differentiated on the basis of their performance on the PPT (a similar but easier test than the Camels and Cactus test used by Barrera et al.), although high scoring in both patients and controls suggests something of a ceiling effect for our cohort. However, our data indicate that impaired semantic processing, possibly at the level of naming, but certainly in relation to lexical access and fluency, is associated with higher levels of TD. Moreover, the observation that semantic fluency differences survive covariance with both current IQ and phonological fluency strengthens the argument that impaired semantic processing is a distinct feature of TD and not simply a consequence of low IQ or impaired phonological fluency.

Our findings are thus broadly congruent with a small but growing body of research evidence linking TD in schizophrenia to a combination of executive dysfunction and circumscribed semantic impairments related to, but possibly not restricted to, access. Earlier studies (Anand et al. 1994; Rodriguez-Ferrera \& McKenna, 1996) hinted at this relationship but were limited by the tests used and/or other methodological constraints. For instance, in the latter study, the authors recorded measures of linguistic and general cognition (though not executive function) in TD patients, concluding that language impairment (where it occurs in schizophrenia) is likely to be a function of a generalized cognitive deficit, which frequently characterizes the disorder. However, in a further study (RodriguezFerrera et al. 2001) the same research group acknowledged that some patients appeared to have specific semantic impairments in the absence of other general cognitive impairments that were associated with TD.

The inclusion by Barrera et al. of a range of established measures of frontal/executive function in addition to tests of semantic and syntactic function has further clarified the neurocognitive signature of TD, and our findings reinforce the conclusions of these researchers; namely, that higher levels of TD in schizophrenia are predicted by poor executive functioning and impaired semantic access, but not by deficits in syntactic processing. Semantic fluency is sub-served by a neural network comprising the left (Pihlajamaki et al. 2000) and right (Billingsley et al. 2004) frontal lobe, and the left medial temporal lobe, which is specifically associated with access to semantic storage (Gourovitch et al. 2000). A positron emission tomography (PET) study of activations related 
to speech production in thought-disordered schizophrenic patients (McGuire et al. 1998) similarly identified cortical (inferior frontal and left superior temporal), cingulate and subcortical (right caudate) regions of irregular activity associated with the production of disordered speech. The idea of dysfunctional neuro-circuitry underpinning TD (and other psychotic symptom domains) has also been elaborated by Jennings et al. (1998), Spence et al. (2000) and recently by Tamminga \& Holcomb (2005), who concluded that the evidence points 'to problems of integration across regions rather than one specific regional abnormality' (p. 31). Although no attempt was made in the present study to delineate crucial neural substrates of TD, our findings in respect of executive and semantic dysfunctions are at least consistent with a growing list of imaging studies in which 'functional disconnectivities' between frontal, temporal and cingulate regions have been linked to TD.

The findings from the present study should be considered in light of several intrinsic methodological limitations. First, in deliberately opting for 'breadth of coverage' in the selection of tests, rather than focusing on performance on a small number of tests in a large sample, our study is underpowered. Second, the absence of significant differences between patients and controls on several of the language tests suggests either that our patients were not particularly impaired or that our tests were not sufficiently sensitive. Third, in common with many other studies of neurocognition in schizophrenia, there is, in the present study, the possibility of a recruitment bias stemming from the fairly demanding nature of some of the tests in our battery, with some patients possibly being judged as 'not competent to complete' them. Additionally, discrepancies remain between our findings and those of Goldberg et al., Rodriguez-Ferrera et al. and Barrera et al. that we are, at present, obliged to account for in terms of the idiosyncrasies of patient recruitment and extraneous variables related to test selection and procedures, and that certainly merit further careful investigation.

In summary, our data provide scant evidence that TD in schizophrenia is directly related to generalized impaired language functioning. TD was not associated with 'semantic inefficiency', or with any of our syntactic measures. However, it was associated with impaired performance on several neurocognitive tests, some of which 'tap' frontal lobe function, and on semantic tests of naming, access and fluency, the latter of which relies on a combination of executive control and semantic access. Further investigation of the relative contributions of executive and semantic impairments to TD in schizophrenia is thus warranted.

\section{DECLARATION OF INTEREST}

None.

\section{REFERENCES}

Ammons, R. B. \& Ammons, C. H. (1962). Quick Test. Psychological Test Specialists: Missoula.

Anand, A., Wales, R. J., Jackson, H. J. \& Copolov, D. L. (1994). Linguistic impairment in early psychosis. Journal of Nervous and Mental Disease 182, 488-493.

Andreasen, N. C. (1979). Thought language and communication disorders. I. Clinical assessment, definition of terms and evaluation of reliability. Archives of General Psychiatry 36, 1315-1321.

Andreasen, N. C. (1987). The Comprehensive Assessment of Symptoms and History. University of Iowa College of Medicine: Iowa City.

APA (1994). Diagnostic and Statistical Manual of Mental Disorders (4th edn). American Psychiatric Association: Washington, DC.

Baddeley, A., Emslie, H. \& Smith, I. N. (1992). The Speed and Capacity of Language Processing Test. Thames Valley: Bury St Edmonds.

Barrera, A., McKenna, P. J. \& Berrios, G. E. (2005). Formal thought disorder in schizophrenia: an executive or a semantic deficit? Psychological Medicine 35, 121-132.

Billingsley, R. L., Simos, P. G., Castillo, E. M., Sarkari, S., Breier, J. I., Pataraia, E. \& Papanicolaou, A. C. (2004). Spatio-temporal cortical dynamics of phonemic and semantic fluency. Journal of Clinical and Experimental Neuropsychology 26, 1031-1043.

Bishop, D. V. M. (1989). Test for the Reception of Grammar (2nd edn). Medical Research Council: London.

Bleuler, E. (1950). Dementia Praecox or the Group of Schizophrenias. International Universities Press: New York.

Coghlan, A. K. (1985). Adult Memory and Information Processing Speed Battery. St James University Hospital: Leeds.

DeRenzi, E. \& Ferrari, C. (1978). The Reporter's Test: a sensitive test to detect expressive disturbances in aphasia. Cortex 14, 279-293.

DeRenzi, E. \& Vignolo, L. A. (1962). The Token Test: a sensitive test to detect disturbances in aphasics. Brain 85, 665-678.

Dunn, L. M. \& Dunn, E. S. (1981). Peabody Picture-Vocabulary Test: Revised. Technical Supplement. American Guidance Service: Circle Pines, MN.

Frith, C. D. (1992). The Cognitive Neuropsychology of Schizophrenia. Lawrence Erlbaum Associates: Hove.

Goldberg, T. E., Dodge, M., Aloia, M., Egan, M. F. \& Weinberger, D. R. (1998). Cognitive substrate of thought disorder. I. The semantic system. American Journal of Psychiatry 155, 1671-1676.

Gourovitch, M. L., Goldberg, T. E. \& Weinberger, D. R. (1996). Verbal fluency deficits in patients with schizophrenia: semantic fluency is differentially impaired as compared to phonological fluency. Neuropsychology 10, 573-577.

Gourovitch, M. L., Kirkby, B. S., Goldberg, T. E., Weinberger, D. R., Gold, J. M., Espositio, G., Van Horn, J. D. \& Berman, K. F. (2000). A comparison of $\mathrm{rCBF}$ patterns during letter and semantic fluency. Neuropsychology 14, 353-360. 
Harrow, M. \& Prossen, M. (1978). Intermingling and disordered logic as influences on schizophrenic 'thought disorders'. Archives of General Psychiatry 35, 1213-1218.

Howard, D. \& Patterson, K. (1992). Pyramids and Palm Trees: A Test of Semantic Access from Pictures and Words. Thames Valley Test Company: Bury St Edmonds.

Jennings, J. M., McIntosh, A. R., Kapur, S., Zipursky, R. B. \& Houle, S. (1998). Functional network differences in schizophrenia: a rCBF study of semantic processing. Neuroreport 9, 1697-1700.

Jones-Gotman, M. \& Milner, B. (1977). Design fluency: the invention of nonsense drawings after focal cortical lesions. Neuropsychologia 15, 653-674

Kay, S. R., Opler, L. A. \& Fisbein, A. (1986). Positive and Negative Syndrome Scale (Manual). Multi-Health Systems: North Tonawanda, NY.

Kerns, J. G. \& Berenbaum, H. (2002). Cognitive impairments associated with formal thought disorder in people with schizophrenia. Journal of Abnormal Psychology 111, 211-224.

McGrath, J. J. (1991). Ordering thoughts on thought disorder. British Journal of Psychiatry 158, 307-316.

McGrath, J. J., Hengstberger, P., Scheldt, S. \& Dark, F. (1997) Thought disorder and executive ability. Cognitive Neuropsychiatry 2, 303-314

McGuire, P. K., Quested, D. J., Spence, S. A., Murray, R. M., Frith, C. D. \& Liddle, P. F. (1998). Pathophysiology of positive thought disorder in schizophrenia. British Journal of Psychiatry 173, 231-235.

McKenna, P. J. \& Warrington, E. (1983). The Graded Naming Test. NFER-Nelson: Windsor, Berks.

Mortimer, A., Corridan, B., Rudge, S., Kho, K., Kelly, F., Bristow, M. \& Hodges, J. (1995). Thought speech and language disorder and semantic memory in schizophrenia. In Speech and Language Disorders in Psychiatry (ed. A. Sims), pp. 70-79. Gaskell: London.

Nelson, H. E. \& O'Connell, A. (1978). Dementia: the estimation of pre-morbid intelligence using the New Adult Reading Test. Cortex 14, 234-244.

Oh, T. M., McCarthy, R. A. \& McKenna, P. J. (2002). Is there a schizophasia? A study applying the single-case approach to formal thought disorder in schizophrenia. Neurocase 8, 233-244.

Pihlajamaki, M., Tanila, H., Hanninen, T., Kononen, M., Laakso, M. Partanen, K., Soininen, H. \& Aronen, H. J. (2000). Verbal fluency activates the left medial temporal lobe: an fMRI study. Annals of Neurology 47, 470-476.

Raven, J. C. (1960). Guide to the Standard Progressive Matrices. H. K. Lewis: London.

Reitan, R. M. (1958). Validity of the Trail Making Test as an indicator of organic brain disease. Perceptual and Motor Skills $\mathbf{8}$, 271-276.

Rodriguez-Ferrera, S., McCarthy, R. A. \& McKenna, P. J. (2001). Language in schizophrenia and its relationship to formal thought disorder. Psychological Medicine 31, 197-205.

Rodriguez-Ferrera, S. \& McKenna, P. J. (1996). Neurolinguistic deficits in schizophrenia are a function of general intellectual impairment and not the presence of formal thought disorder. Schizophrenia Research 18, 207.

Rossell, S. L., Rabe-Hesketh, S. S., Shapleke, J. S. \& David, A. S. (1999). Is semantic fluency differentially impaired in schizophrenic patients with delusions? Journal of Clinical and Experimental Psychology 21, 629-642.

Shallice, T. (1982). Specific impairments of planning. Philosophical Transactions of the Royal Society of London 298, 199-209.

Spence, S. A., Liddle, P. F., Stefan, M. D., Hellewell, J. S. E., Sharma, T., Friston, K. J., Hirsch, S. R., Frith, C. D., Murray R. M., Deakin, J. F. W. \& Grasby, P. M. (2000). Functional anatomy of verbal fluency in people with schizophrenia and those at genetic risk. British Journal of Psychiatry 176, 52-60.

Spreen, O. \& Benton, A. L. (1969). Neurosensory Centre Comprehensive Examination for Aphasia. Neuropsychology Laboratory, Department of Psychology, University of Victoria: Victoria, BC.

Stirling, J. D., Hellewell, J. S. E. \& Hewitt, J. (1997). Verbal memory impairment in schizophrenia: no sparing of short term recall. Schizophrenia Research 25, 85-95.

Stroop, J. R. (1935). Studies of interference in serial verbal reactions. Journal of Experimental Psychology 18, 643-662.

Tamlyn, D., McKenna, P. J., Mortimer, A. M., Lund, C. E. Hammond, S. \& Baddeley, A. D. (1992). Memory impairment in schizophrenia: its extent, affiliations and neuropsychological character. Psychological Medicine 22, 101-115.

Tamminga, C. A. \& Holcomb, H. H. (2005). Phenotype of schizophrenia: a review and formulation. Molecular Psychiatry 10, 27-39.

Wechsler, D. (1987). Wechsler Memory Scale Revised. Harcourt Brace Jovanovich: San Antonio. 